\title{
Violation of Entropic Leggett-Garg Inequality in Nuclear Spins
}

\author{
Hemant Katiyar ${ }^{1}$, Abhishek Shukla ${ }^{1}$, Rama Koteswara Rao ${ }^{2}$, and T. S. Mahesh ${ }^{1 *}$ \\ ${ }^{1}$ Department of Physics and NMR Research Center, \\ Indian Institute of Science Education and Research, Pune 411008, India \\ ${ }^{2}$ Department of Physics and NMR Research Center, Indian Institute of Science, Bangalore, India
}

\begin{abstract}
We report an experimental study of recently formulated entropic Leggett-Garg inequality (ELGI) by Usha Devi et al. (arXiv: 1208.4491v2 (2012)). This inequality places a bound on the statistical measurement outcomes of dynamical observables describing a macrorealistic system. Such a bound is not necessarily obeyed by quantum systems, and therefore provides an important way to distinguish quantumness from classical behavior. Here we study ELGI using a two-qubit nuclear magnetic resonance system. To perform the noninvasive measurements required for the ELGI study, we prepare the system qubit in a maximally mixed state as well as use the 'ideal negative result measurement' procedure with the help of an ancilla qubit. The experimental results show a clear violation of ELGI by over four standard deviations. These results agree with the predictions of quantum theory. The violation of ELGI is attributed to the fact that certain joint probabilities are not legitimate in the quantum scenario, in the sense they do not reproduce all the marginal probabilities. Using a three-qubit system, we experimentally demonstrate that three-time joint probabilities do not reproduce certain two-time marginal probabilities.
\end{abstract}

PACS numbers: 03.67.Lx,03.65.Ta, 03.67.Ac, 76.30.-v

Keywords: Leggett-Garg Inequality, Shannon Entropy, Joint Probabilities

Introduction. - The behavior of quantum systems is often incomprehensible by classical notions, the best examples being nonlocality $[1,2]$ and contextuality [3]. Quantum systems are nonlocal since they violate Bell's inequality, which assumes that local operations on one of the two space-like separated objects can not disturb the measurement outcomes of the other [4]. The quantum systems are also contextual in the sense that a measurement outcome depends not only on the system and the property being measured, but also on the context of the measurement, i.e., on the set of other compatible properties which are being measured along with.

Another notion imposed on classical objects is macrorealism, which is based on two criteria: (i) the object remains in one or the other of many possible states at all times, and (ii) the measurements are noninvasive, i.e., they reveal the state of the object without disturbing the object or its future dynamics. Quantum systems are incompatible with these criteria and therefore violate bounds on correlations derived from them. For instance, Leggett-Garg inequality (LGI) sets up macrorealistic bounds on linear combinations of two-time correlations of a dichotomic observable belonging to a single dynamical system [5]. In this sense, LGI is considered as a temporal analogue of Bell's inequality. Quantum systems do not comply with LGI, and therefore provide an important way to distinguish the quantum behavior from macrorealism. Violations of LGI by quantum systems have been investigated and demonstrated experimentally in various systems $[6-9]$.

For understanding the quantum behavior it is important to investigate it through different approaches, particularly from an information theoretical point of view. For example, an entropic formulation for Bell's inequality has been given by Braunstein and Caves [10], and more recently that for contextuality has been given independently by Rafael and Fritz [11] and Kurzyński et.al. [12]. Recently, an entropic formulation of LGI has also been introduced by Usha Devi et al. [13], in terms of classical Shannon entropies associated with classical correlations. Such entropies obey certain constraints, which when violated would imply non-existence of legitimate joint probabilities (JP) for all the measurement outcomes.

Here we report an experimental demonstration of violation of entropic LGI (ELGI) in an ensemble of spin $1 / 2$ nuclei using nuclear magnetic resonance (NMR) techniques. Although NMR experiments are carried out at a high temperature limit, the nuclear spins have long coherence times, and their unitary evolutions can be controlled in a precise way. The large parallel computations carried out in an NMR spin ensemble assists in efficiently extracting the single-event probability (SEP) and JP. The simplest ELGI study involves three sets of twotime joint measurements of a dynamic observable belonging to a 'system' qubit at time instants $\left(t_{1}, t_{2}\right),\left(t_{2}, t_{3}\right)$, and $\left(t_{1}, t_{3}\right)$. The first measurement in each case must be 'noninvasive' in the sense, it should not influence the outcome of the second measurement. These noninvasive measurements (NIM) can be performed with the help of an ancilla qubit.

Further, it has been argued in [13] that the violation of ELGI arises essentially due to the fact that the JP do not originate from a legitimate grand probability (of which the JP are the marginals). Here we describe extracting three-time JP using a three-qubit system, and demonstrate experimentally that it can not reproduce all the marginal probabilities (MP) substantiating this feature.

In the following we briefly revisit the theory of ELGI 
[13] and then we describe the circuits for the measurement of SEP and JP. Later we detail its experimental study using a two-qubit NMR system. Then we describe the study of three-time JP using a three-qubit NMR system.

Theory. - Consider a dynamical observable $Q\left(t_{k}\right)=$ $Q_{k}$ measured at different time instances $t_{k}$. Let the measurement outcomes be $q_{k}$ with probabilities $P\left(q_{k}\right)$. In classical information theory, the amount of information stored in the random variable $Q_{k}$ is given by the Shannon entropy [14],

$$
H\left(Q_{k}\right)=-\sum_{q_{k}} P\left(q_{k}\right) \log _{2} P\left(q_{k}\right) .
$$

The conditional information stored in $Q_{k+l}$ at time $t_{k+l}$, assuming that the observable $Q_{k}$ has an outcome $q_{k}$, is

$$
H\left(Q_{k+l} \mid Q_{k}=q_{k}\right)=-\sum_{q_{k+l}} P\left(q_{k+l} \mid q_{k}\right) \log _{2} P\left(q_{k+l} \mid q_{k}\right),
$$

where $P\left(q_{k+l} \mid q_{k}\right)$ is the conditional probability. Then the mean conditional entropy is given by,

$$
H\left(Q_{k+l} \mid Q_{k}\right)=-\sum_{q_{k}} P\left(q_{k}\right) H\left(Q_{k+l} \mid Q_{k}=q_{k}\right) .
$$

Using Bayes' theorem, $P\left(q_{k+l} \mid q_{k}\right) P\left(q_{k}\right)=P\left(q_{k+l}, q_{k}\right)$, the mean conditional entropy becomes

$$
H\left(Q_{k+l} \mid Q_{k}\right)=H\left(Q_{k}, Q_{k+l}\right)-H\left(Q_{k}\right),
$$

where the joint Shannon entropy is given by

$$
H\left(Q_{k}, Q_{k+l}\right)=-\sum_{q_{k}, q_{k+l}} P\left(q_{k+l}, q_{k}\right) \log _{2} P\left(q_{k+l}, q_{k}\right) .(4)
$$

These Shannon entropies always follow the inequality [10]

$$
H\left(Q_{k+l} \mid Q_{k}\right) \leq H\left(Q_{k+l}\right) \leq H\left(Q_{k}, Q_{k+l}\right) .
$$

The left side of the equation implies that removing a constraint never decreases the entropy, and the right side implies information stored in two variables is always greater than or equal to that in one [13]. Suppose that three measurements $Q_{k}, Q_{k+l}$, and $Q_{k+m}$, are performed at time instants $t_{k}<t_{k+l}<t_{k+m}$. Then, from equations (3) and (5), the following inequality can be obtained:

$$
H\left(Q_{k+m} \mid Q_{k}\right) \leq H\left(Q_{k+m} \mid Q_{k+l}\right)+H\left(Q_{k+l} \mid Q_{k}\right) .
$$

For $n$ measurements $Q_{1}, Q_{2}, \ldots, Q_{n}$, at time instants $t_{1}<t_{2}<\cdots<t_{n}$, the above inequality can be generalized to [13]

$$
\sum_{k=2}^{n} H\left(Q_{k} \mid Q_{k-1}\right)-H\left(Q_{n} \mid Q_{1}\right) \geq 0 .
$$

This inequality must be followed by all macro-realistic objects, since its satisfaction means the existence of le- gitimate JP distribution, which can yield all MP [12].

Usha Devi et al. [13] have shown theoretically that the above inequality is violated by a quantum spin- $s$ system, prepared in a completely mixed initial state, $\rho_{\text {in }}=\mathbb{1} /(2 s+1)$. Consider the $z$-component of the spin evolving under the Hamiltonian $\mathcal{H}=-\omega S_{x}$ as our dynamical observable, i.e. $Q_{t}=U_{t} S_{z} U_{t}^{\dagger}$, where $U_{t}=e^{-i \mathcal{H} t}$, and $S_{x}$ and $S_{z}$ are the components of spinangular momentum. Let $n$-measurements occur at regular time instants $\Delta t, 2 \Delta t, \cdots, n \Delta t$. Ideally in this case, the conditional entropies $H\left(Q_{k} \mid Q_{k-1}\right)$ between successive measurements are all equal, and can be denoted as $H[\theta /(n-1)]$, where $\theta /(n-1)=\omega \Delta t$ is the rotation caused by the Hamiltonian in the interval $\Delta t$. Similarly we can denote $H\left(Q_{n} \mid Q_{1}\right)$ as $H[\theta]$. The lhs of inequality (7) scaled in units of $\log _{2}(2 s+1)$ is termed as the information deficit $\mathcal{D}$. For $n$-equidistant measurements, it can be written as [13]

$$
\mathcal{D}_{n}(\theta)=\frac{(n-1) H[\theta /(n-1)]-H[\theta]}{\log _{2}(2 s+1)} \geq 0 .
$$

Measurement of Probabilities. - Consider a spin- $1 / 2$ particle as the system qubit. Using the eigenvectors $\{|0\rangle,|1\rangle\}$ of $S_{z}$, as the computational basis, the projection operators at time $t=0$ are $\left\{\Pi_{\alpha}=|\alpha\rangle\langle\alpha|\right\}_{\alpha=0,1}$. For the dynamical observable, the measurement basis is rotating under the unitary $U_{t}=e^{i \omega S_{x} t}$, such that $\Pi_{\alpha}^{t}=U_{t} \Pi_{\alpha} U_{t}^{\dagger}$. However, it is convenient to perform the actual measurements in the time-independent computational basis. Since for an instantaneous state $\rho(t)$, $\Pi_{\alpha}^{t} \rho(t) \Pi_{\alpha}^{t}=U_{t} \Pi_{\alpha}\left(U_{t}^{\dagger} \rho(t) U_{t}\right) \Pi_{\alpha} U_{t}^{\dagger}$, measuring in $\left\{\Pi_{\alpha}^{t}\right\}$ basis is equivalent to back-evolving the state by $U_{t}^{\dagger}$, measuring in computational basis, and lastly forward evolving by $U_{t}$. This latter evolution can be omitted if one is interested only in the probabilities and not in the post measurement state of the system. For example, in case of multiple-time measurements, the forward evolution can be omitted after the final measurement.

The method for extracting SEP and JP involves the quantum circuits shown in Fig. 1. To measure SEP $P\left(q_{i}\right)$ of system qubit in a general state $\rho_{S}$, it is evolved by $U_{i}^{\dagger}=e^{i \mathcal{H} t_{i}}$, and the probabilities $P\left(q_{i}\right)$ are obtained using diagonal tomography (Fig. 1(a)). Here a further forward evolution by $U_{i}$ is not necessary as described earlier.

To measure JP $P\left(q_{i}, q_{j}\right)$, we utilize an ancilla qubit initialized in the state $|0\rangle\langle 0|$ (Fig. 1(b)). After back evolution to computational basis, the CNOT gate encodes the probabilities of the system-qubit $P\left(q_{i}\right)$ on to the ancilla-qubit [15]. After a further evolution by $U_{i} U_{j}^{\dagger}=e^{-i \omega S_{x}\left(t_{j}-t_{i}\right)}$, a diagonal tomography of the two qubit system yields $P\left(q_{i}, q_{j}\right)$ [15].

A similar scheme, shown in Fig. 1(c), is employed for extracting three-time JP. These circuits can be general- 


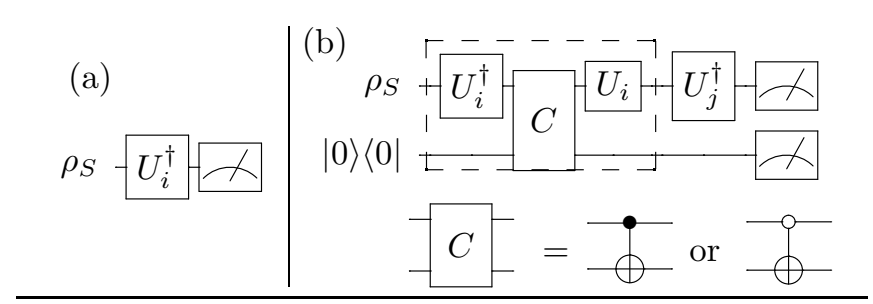

(c)

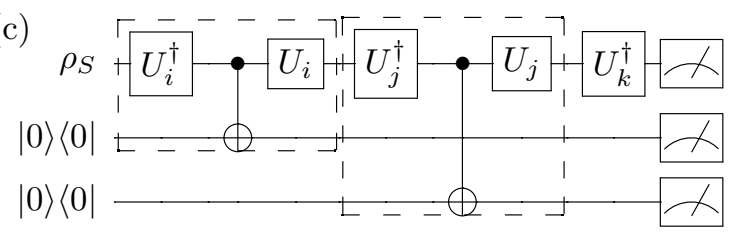

FIG. 1. Circuits for measuring SEP (a), two-time JP (b), and three-time JP (c). The grouped gates represent measurement in $\left\{\Pi_{0}^{t}, \Pi_{1}^{t}\right\}$ basis. In (b) the operation $C$ can be either CNOT or anti-CNOT gate as described in the text.

ized for higher order JP or for spin- $s>1 / 2$ systems, using appropriate ancilla register.

In the earlier LGI experiments, NIM have been performed by either (i) a weak measurement which causes minimum disturbance to the quantum state [7] or (ii) initializing the system qubit in a maximally mixed state so that the system density matrix remains unchanged by the measurements [8]. Recently however, it was noted by Knee et al. that a sceptical macrorealist is not convinced by either of the above methods [16]. Instead, they had proposed a 'ideal negative result measurement' (INRM) procedure that is more convincingly noninvasive [9]. The idea is as follows. The CNOT gate is able to flip the ancilla qubit only if the system qubit is in state $|1\rangle$, and does nothing if the system qubit is in state $|0\rangle$. Therefore after the CNOT gate, if we measure the probability of unflipped ancilla, this corresponds to an 'interactionfree' or NIM of $P(q=0)$. Similarly, we can implement an anti-CNOT gate, which flips the ancilla only if the system qubit is in state $|0\rangle$, and does nothing otherwise, such that the probability of unflipped qubit now gives $P(q=1)$. Note that in both the cases, the probabilities wherein the system interacted with the ancilla, resulting in its flip, are discarded. The final measurement need not be NIM since we are not concerned about any further evolution.

In our experiments we combine the two methods, i.e., (i) first we prepare the system in a maximally mixed state i.e., $\rho_{S}=\mathbb{1} / 2$, and (ii) we perform INRM. In this case, $P\left(0_{i}\right)=P\left(1_{i}\right)=1 / 2$, and $\mathrm{JP}$ are

$$
\begin{aligned}
& P\left(0_{i}, 0_{j}\right)=\left|\cos \left(\theta_{i j} / 2\right)\right|^{2} / 2=P\left(1_{i}, 1_{j}\right), \quad \text { and }, \\
& P\left(0_{i}, 1_{j}\right)=\left|\sin \left(\theta_{i j} / 2\right)\right|^{2} / 2=P\left(1_{i}, 0_{j}\right),
\end{aligned}
$$

where $\theta_{i j}=\omega\left(t_{j}-t_{i}\right)[13]$.

The only single event entropy needed for the ELGI test is $H\left(Q_{1}\right)$, since $H\left(Q_{t}\right)$ is constant for the maximally mixed system state. Further, since $H\left(Q_{1}, Q_{2}\right)=$
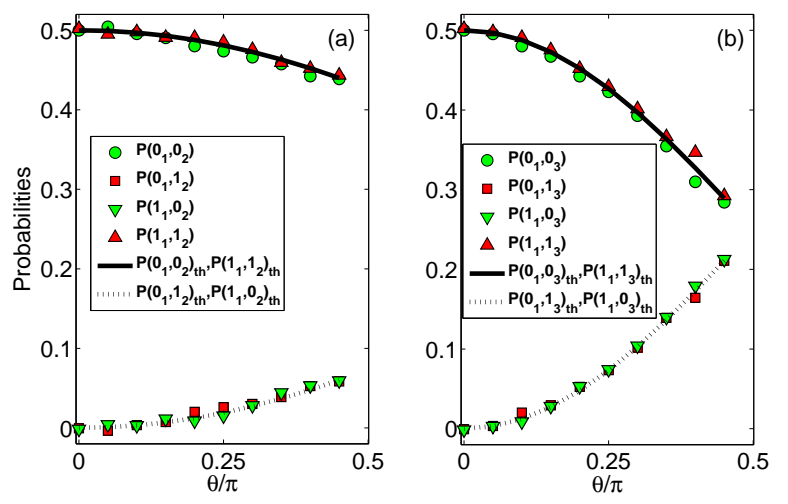

FIG. 2. The lines indicate theoretical JP and the symbols indicate the mean experimental probabilities obtained by INRM procedure.

$H\left(Q_{2}, Q_{3}\right)$ in the case of uniform time intervals, only two joint entropies $H\left(Q_{1}, Q_{2}\right)$ and $H\left(Q_{1}, Q_{3}\right)$ are needed to be measured for evaluating $\mathcal{D}_{3}$. In the following we describe the experimental implementation of these circuits for the three-measurement LGI test.

Experiment. - We have used ${ }^{13} \mathrm{CHCl}_{3}$ (dissolved in $\mathrm{CDCl}_{3}$ ) as the two qubit system and treat its ${ }^{13} \mathrm{C}$ and ${ }^{1} \mathrm{H}$ nuclear spins as the system and the ancilla qubits respectively. The resonance offset of ${ }^{13} \mathrm{C}$ was set to $100 \mathrm{~Hz}$ (provides a dynamic 'observable') and that of ${ }^{1} \mathrm{H}$ to $0 \mathrm{~Hz}$ (on resonant). The two spins have an indirect spin-spin coupling constant $J=209.2 \mathrm{~Hz}$ [15]. All the experiments were carried out at an ambient temperature of $300 \mathrm{~K}$ on a $500 \mathrm{MHz}$ Bruker UltraShield NMR spectrometer.

The evolution propagator $U j^{\dagger} U_{i}=e^{-i S_{x} \omega\left(t_{j}-t_{i}\right)}$ is realized by the cascade $\mathbb{H} U_{d} \mathbb{H}$, where $\mathbb{H}$ is the Hadamard gate, and the delay propagator $U_{d}=e^{-i S_{z} \omega\left(t_{j}-t_{i}\right)}$ corresponds to the $\hat{z}$-precession of the system qubit at $\omega=2 \pi 100 \mathrm{rad} / \mathrm{s}$ resonance off-set. The $J$-evolution during this delay is refocused by a $\pi$ pulse on the ancilla qubit. The CNOT, $\mathbb{H}$, as well as the $\pi$ pulses are realized by numerically optimized amplitude and phase modulated $\mathrm{RF}$ pulses, and are robust against the RF inhomogeneity with a average Hilbert-Schmidt fidelity better than 0.998 [17-19]. The final measurement of probabilities are carried out by diagonal tomography. It involved dephasing all the coherences using a strong pulsed field gradient followed by a $\pi / 30$ detection pulse. The intensities of the resulting spectral lines yielded a traceless diagonal density matrix, which was normalized and added with identity matrix to extract the probabilities. As described in Fig. 1(b), two sets of experiments were performed, one with CNOT and the other with anti-CNOT. We extracted $P(0, q)(q=\{0,1\})$ from the CNOT set, and $P(1, q)$ from the anti-CNOT set. The probabilities thus obtained by INRM procedure are plotted in Fig. 2 . These sets of experiments also allow us to compare the 

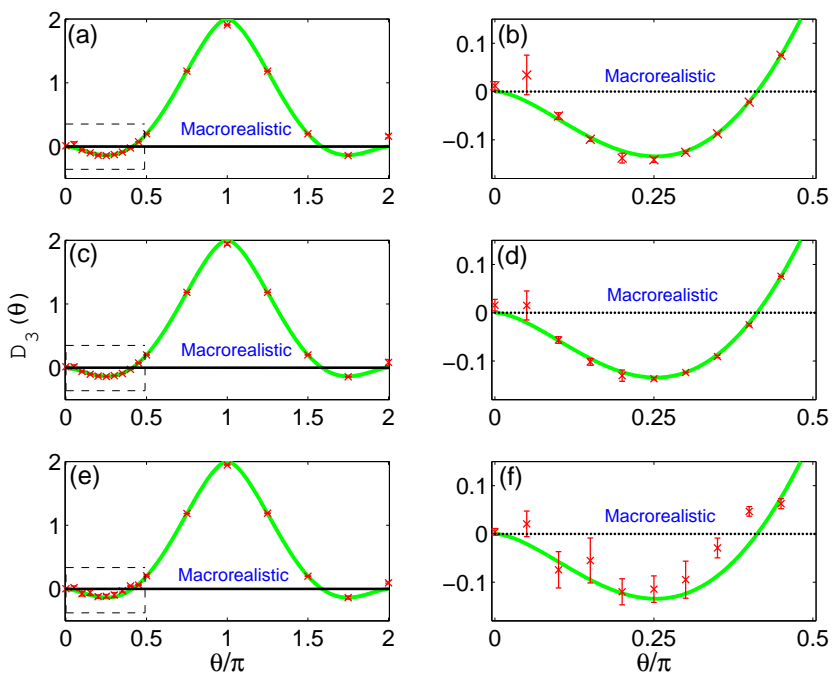

FIG. 3. Information deficit $\mathcal{D}_{3}$ versus $\theta$ obtained with CNOT (a,b), anti-CNOT (c,d), and INRM procedure (e,f). The boxed areas in the left plots (a,c,e) are magnified in the right plots (b,d,f) respectively. The mean experimental $\mathcal{D}_{3}$ (in bits) values are shown as symbols. The curves indicate theoretical $\mathcal{D}_{3}$ (in bits). The horizontal line $\mathcal{D}_{3}=0$ indicates the lower bound of the macrorealism territory.

results from (i) only CNOT, (ii) only anti-CNOT, and (iii) INRM procedures. The joint entropies were calculated in each case using the experimental probabilities and the information deficit (in bits) was calculated using the expression $\mathcal{D}_{3}=2 H\left(Q_{2} \mid Q_{1}\right)-H\left(Q_{3} \mid Q_{1}\right)$. The theoretical and experimental values of $\mathcal{D}_{3}$ for various rotation angles $\theta$ are shown in Fig. 3. We find a general agreement between the mean experimental $\mathcal{D}_{3}$ values with that of the quantum theory. The error bars indicate the standard deviations obtained by a series of independent measurements. According to quantum theory, a maximum violation of $\mathcal{D}_{3}=-0.134$ should occur at $\theta=\pi / 4$. The experimental values of $\mathcal{D}_{3}(\pi / 4)$ are $-0.141 \pm 0.005,-0.136 \pm 0.002$, and $-0.114 \pm 0.027$ for the CNOT, anti-CNOT, and INRM cases respectively. Thus in all the cases, we found a clear violation of ELGI.

Three-time JP. - It has been argued that the violation of ELGI in quantum systems is essentially because certain JP can not reproduce all MP [13]. For example, in the $\mathcal{D}_{3}$ experiment described earlier, the two-time JP $P\left(q_{1}, q_{2}\right), P\left(q_{2}, q_{3}\right)$, and $P\left(q_{1}, q_{3}\right)$ can be obtained from eqns. (9). From the three-time JP, it is possible to generate MP:

$$
\begin{aligned}
& P^{\prime}\left(q_{1}, q_{2}\right)=\sum_{q_{3}} P\left(q_{1}, q_{2}, q_{3}\right), \\
& P^{\prime}\left(q_{2}, q_{3}\right)=\sum_{q_{1}} P\left(q_{1}, q_{2}, q_{3}\right), \text { and } \\
& P^{\prime}\left(q_{1}, q_{3}\right)=\sum_{q_{2}} P\left(q_{1}, q_{2}, q_{3}\right) .
\end{aligned}
$$

Now $P\left(q_{1}, q_{2}, q_{3}\right)$ can reproduce $P\left(q_{1}, q_{2}\right)$ and $P\left(q_{2}, q_{3}\right)$, i.e., $P^{\prime}\left(q_{1}, q_{2}\right)=P\left(q_{1}, q_{2}\right)$ and $P^{\prime}\left(q_{2}, q_{3}\right)=P\left(q_{2}, q_{3}\right)$. However, $P\left(q_{1}, q_{2}, q_{3}\right)$ can not reproduce $P\left(q_{1}, q_{3}\right)$, i.e., $P^{\prime}\left(q_{1}, q_{3}\right) \neq P\left(q_{1}, q_{3}\right)$, in general [13].

The above concept can be investigated experimentally by measuring the three-time JP, as described in Fig. 1(c). Since this experiment requires measurements at three time instants, we need two ancilla qubits along with the system qubit. We use the three ${ }^{19} \mathrm{~F}$ nuclear spins (spin$1 / 2$ ) of trifluoroiodoethylene dissolved in acetone-D6 as the three-qubit system [15]. The chemical shifts differences are $\nu_{2}-\nu_{1}=11860.8 \mathrm{~Hz}, \nu_{2}-\nu_{3}=17379.1 \mathrm{~Hz}$, and the scalar coupling constants are: $J_{12}=69.9 \mathrm{~Hz}$, $J_{13}=-128.3 \mathrm{~Hz}$, and $J_{23}=47.4 \mathrm{~Hz}$. Here the first spin $\left(\mathrm{F}_{1}\right)$ is used as the system qubit and the others $\left(F_{2}\right.$ and $\left.F_{3}\right)$ are chosen as the ancilla qubits. Initialization involved preparing the state, $\frac{1-\epsilon}{8} \mathbb{1}+\epsilon\left\{\frac{1}{2} \mathbb{1}_{S} \otimes|00\rangle\left\langle\left. 00\right|_{A}\right\}\right.$ where $\epsilon \sim 10^{-5}$ is the purity factor $[15,20]$. The experimental three-time JP $P\left(q_{1}, q_{2}, q_{3}\right)$ obtained using the circuit Fig. 1(c) are shown in Fig. 4(a). Two-time JP $P\left(q_{i}, q_{j}\right)$ were also obtained using a similar circuit (Fig. $1(\mathrm{c})$ without $\left.U_{j} U_{k}^{\dagger}\right)$. Here JP are completely stored in the ancilla qubits and were obtained by tracing out the system qubit. The results $P\left(q_{1}, q_{2}\right), P\left(q_{2}, q_{3}\right)$, and $P\left(q_{1}, q_{3}\right)$ are shown in Fig. 4 (b-d). In each plots, we have over-
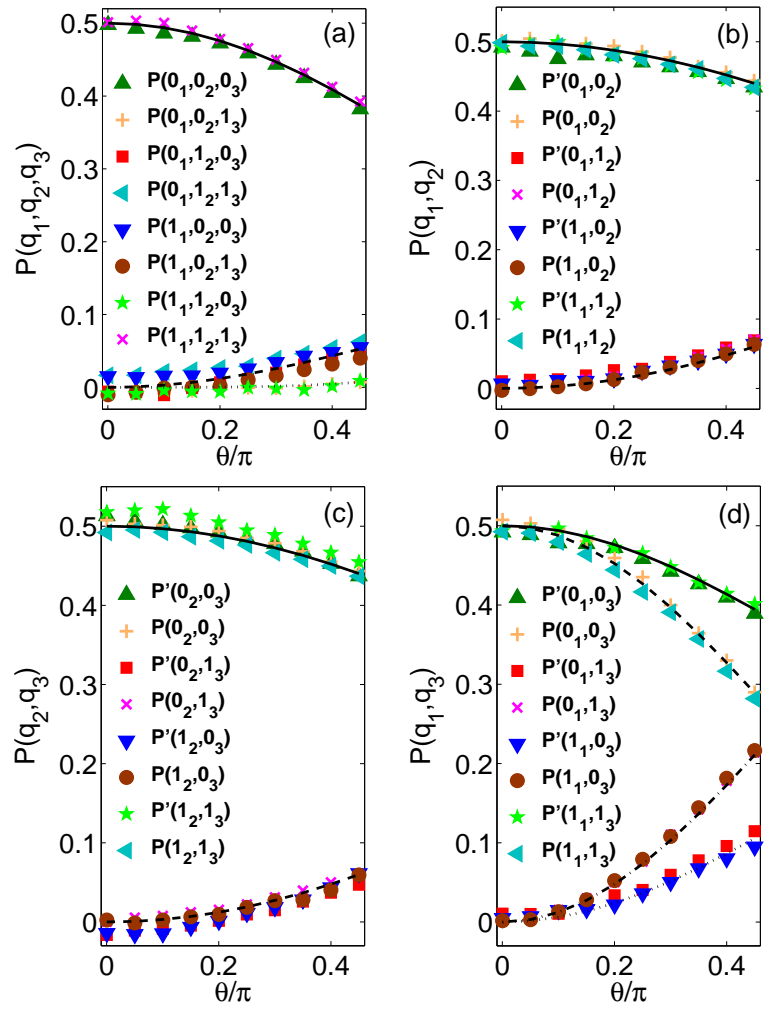

FIG. 4. The three-time JP $P\left(q_{1}, q_{2}, q_{3}\right)$ (a), and two-time JP $P\left(q_{i}, q_{j}\right)$ and MP $P^{\prime}\left(q_{i}, q_{j}\right)$ generated from $P\left(q_{1}, q_{2}, q_{3}\right)$ (b-d). The lines correspond to theoretical values and the symbols are mean experimental values. 
layed MP $P^{\prime}\left(q_{1}, q_{2}\right), P^{\prime}\left(q_{2}, q_{3}\right)$, and $P^{\prime}\left(q_{1}, q_{3}\right)$ generated from the experimental three-time JP. As expected, MP $P^{\prime}\left(q_{1}, q_{2}\right)$ and $P^{\prime}\left(q_{2}, q_{3}\right)$ match well with the JP $P\left(q_{1}, q_{2}\right)$ and $P\left(q_{2}, q_{3}\right)$ respectively, while the $\mathrm{MP} P^{\prime}\left(q_{1}, q_{3}\right)$ does not match with JP $P\left(q_{1}, q_{3}\right)$. These results confirm that the three-time JP $P\left(q_{1}, q_{2}, q_{3}\right)$ is not legitimate in the quantum case. It is interesting to note that even for those values of $\theta$ for which $\mathcal{D}_{3}$ is positive, the three-time JP is illegitimate. Therefore, while the violation of ELGI indicates the quantumness of the system, its satisfaction does not rule out the quantumness.

Conclusions. - We described an experimental study of the entropic Leggett-Garg inequality in nuclear spins using NMR techniques. We employed the recently described 'ideal negative result measurement' procedure to noninvasively extract joint probabilities. Our results indicate the macrorealistic bound being violated by over four standard deviations, confirming the nonmacrorealistic nature of the spin-1/2 particles. Quantum systems do not have legitimate joint probability distribution, which results in the violation of bounds set-up for macrorealistic systems. We have experimentally measured the three-time joint probabilities and confirmed that the two-time joint probabilities are not reproduced as marginals.

One distinct feature of the entropic LGI is that, the dichotomic nature of observables assumed in the original formulation of LGI can be relaxed, thus allowing one to study the quantum behavior of higher dimensional systems such as spin $>1 / 2$ systems. This could be an interesting topic for future experimental investigations.

The authors are grateful to Prof. Usha Devi, Prof. A. K. Rajagopal, Dr. G. C. Knee, Prof. Anil Kumar, Dr. V. Athalye, and S. S. Roy for discussions. This work was partly supported by the DST project SR/S2/LOP$0017 / 2009$.

* mahesh.ts@iiserpune.ac.in

[1] A. Einstein, B. Podolsky, and N. Rosen, Phys. Rev. 47, 777 (1935).

[2] J. Bell, Physics 1, 195 (1964).

[3] S. Kochen and E. P. Specker, J. Math. Mech. 17, 59 (1967).

[4] J. S. Bell, Rev. Mod. Phys. 38, 447 (1966).

[5] A. J. Leggett and A. Garg, Phys. Rev. Lett. 54, 857 (1985).

[6] N. Lambert, R. Johansson, and F. Nori, Phys. Rev B 84, 245421 (2001); J.-S. Xu et al., Sci. Rep. 1, 101 (2011); C. Emary, Phys. Rev B 86, 085418 (2012); Y. Suzuki, M. Iinuma, and H. F. Hofmann, New J. Phys. 14, 103022 (2012); Y.-N. Sun et al., (2012), arXiv:1110.5537; Z.-Q. Zhou et al., (2012), arXiv:1209.2176.

[7] A. Palacios-Laloy et al., Nature Phys. 6, 442 (2010); M. E. Goggin et al.,
Proc. Natl. Acad. Sci. USA 108, 1256 (2011); J. Dressel et al., Phys. Rev. Lett. 106, 040402 (2011).

[8] V. Athalye, S. S. Roy, and T. S. Mahesh, Phys. Rev. Lett. 17, 130402 (2011); A. M. Souza, I. S. Oliveira, and R. S. Sarthour, New J. Phy. 13, 053023 (2011).

[9] G. C. Knee et al., Nat. Commun. 3, 606 (2012).

[10] S. L. Braunstein and C. M. Caves, Phys. Rev. Lett. 61, 662 (1988).

[11] R. Chaves and T. Fritz, Phys. Rev. A 85, 032113 (2012).

[12] P. Kurzyński, R. Ramanathan, and D. Kaszlikowski, Phys. Rev. Lett. 109, 020404 (2012).

[13] A. R. Usha Devi et al., (2012), arXiv:1208.4491.

[14] M. A. Nielsen and I. L. Chuang, Quantum Computation and Quantum Information (Cambridge University Press, 1994).

[15] See supplementary material.

[16] G. C. Knee et al., New J. Phys.. 14, 058001 (2012).

[17] E. M. Fortunato et al., J. Chem. Phys. 116, 7599 (2002).

[18] T. Mahesh and D. Suter, Phys. Rev. A 74, 062312 (2006).

[19] N. Khaneja et al., J. Magn. Reson. 172, 296 (2005).

[20] D. Cory, M. Price, and T. Havel, Physica D 120, 82 (1998). 


\section{SUPPLEMENTARY INFORMATION FOR "VIOLATION OF ENTROPIC LEGGETT-GARG INEQUALITY IN NUCLEAR SPINS"}

Encoding the probability using a CNOT gate: Consider a system qubit initially prepared in a general state $\rho_{S}$ and an ancilla qubit prepared in the state $|0\rangle\langle 0|$. The state of the system qubit can be written as $P\left(0_{i}\right)|0\rangle\langle 0|+$ $P\left(1_{i}\right)|1\rangle\langle 1|+a| 1\rangle\left\langle 0\left|+a^{\dagger}\right| 0\right\rangle\langle 1|$, where $a$ is a complex probability amplitude. The CNOT gate encodes the probability of the outcomes in the diagonal elements of ancilla qubit since,

$$
\begin{array}{r}
\left(P ( 0 _ { i } ) | 0 \rangle \langle 0 | _ { S } + P ( 1 _ { i } ) | 1 \rangle \langle 1 | _ { S } + a | 1 \rangle \langle 0 | _ { S } + a ^ { \dagger } | 0 \rangle \langle 1 | _ { S } ) \otimes | 0 \rangle \langle 0 | _ { A } \stackrel { \mathrm { CNOT } } { \longrightarrow } | 0 \rangle \langle 0 | _ { S } \otimes P ( 0 _ { i } ) | 0 \rangle \langle 0 | _ { A } + | 1 \rangle \langle 1 | _ { S } \otimes P ( 1 _ { i } ) | 1 \rangle \left\langle\left.1\right|_{A}\right.\right. \\
+|1\rangle\left\langle\left. 0\right|_{S} \otimes a \mid 1\right\rangle\left\langle\left. 0\right|_{A}+\mid 0\right\rangle\left\langle\left. 1\right|_{S} \otimes a^{\dagger} \mid 0\right\rangle\left\langle\left. 1\right|_{A} .\right.
\end{array}
$$

The probabilities $P\left(0_{i}\right)$ and $P\left(1_{i}\right)$ can now be retrieved by tracing over the system qubit and reading the diagonal elements of the ancilla state.

The qubit systems: Fig. 5 shows the molecular structures and the Hamiltonian parameters of ${ }^{13} \mathrm{CHCl}_{3}(\mathrm{Fig}$. $5(\mathrm{a}, \mathrm{b})$ ) and trifluoroiodoethylene (Fig. $5(\mathrm{c}, \mathrm{d})$ ). In the case of ${ }^{13} \mathrm{CHCl}_{3}$, spin-lattice (T1) and spin-spin (T2) relaxation time constants for the ${ }^{1} \mathrm{H}$ spin are, respectively, $4.1 \mathrm{~s}$ and $4.0 \mathrm{~s}$. The corresponding time constants for ${ }^{13} \mathrm{C}$ are $5.5 \mathrm{~s}$ and $0.8 \mathrm{~s}$. In the case of trifluoroiodoethylene, the effective ${ }^{19} \mathrm{~F}$ transverse relaxation time constants $\left(T_{2}^{*}\right)$ were about $0.8 \mathrm{~s}$ and their longitudinal relaxation time constants were all longer than $6.3 \mathrm{~s}$.

(a)

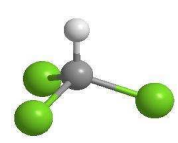

(c)

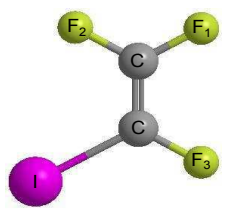

(b)

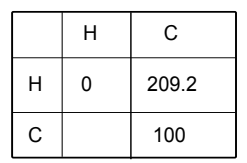

(d)

\begin{tabular}{|c|c|c|c|}
\hline & $F_{1}$ & $F_{2}$ & $F_{3}$ \\
\hline$F_{1}$ & 100.0 & 69.9 & -128.3 \\
\hline$F_{2}$ & & 11960.8 & 47.4 \\
\hline$F_{3}$ & & & -17279.1 \\
\hline
\end{tabular}

(e)

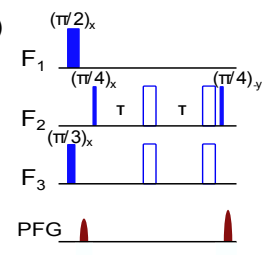

FIG. 5. The molecular structures of chloroform (a) and trifluoroiodoethylene (c) and the corresponding tables (b and d) of relative resonance frequencies (diagonal elements) and the J-coupling constants. The pulse sequence for initializing trifluoroiodoethylene is shown in (e). In (e) the open pulses are $\pi$ pulses and the delay $\tau=1 /\left(4 J_{23}\right)$.

Initialization in two-qubit $\left({ }^{13} \mathbf{C H C l}_{3}\right)$ system: The initialization involved preparing the maximally mixed state $\rho_{S}=\mathbb{1} / 2$ on the system qubit $\left({ }^{13} \mathrm{C}\right)$. This is achieved by a $\pi / 2$ pulse on ${ }^{13} \mathrm{C}$ followed by a strong Pulsed Field Gradient (PFG).

Initialization in three-qubit (trifluoroiodoethylene) system: The equilibrium deviation density matrix evolves under the PPS sequence (Fig. 5(e)) as follows

$$
\begin{aligned}
& S_{1 z}+S_{2 z}+S_{3 z} \stackrel{(\pi / 2)_{1 x}(\pi / 3)_{3 x}, \mathrm{PFG}}{\longrightarrow} S_{z}^{2}+\frac{1}{2} S_{3 z} \stackrel{(\pi / 4)_{2 x}}{\longrightarrow} \frac{1}{\sqrt{2}} S_{2 z}-\frac{1}{\sqrt{2}} S_{2 y}+\frac{1}{2} S_{3 z} \stackrel{1 /\left(2 J_{23}\right)}{\longrightarrow} \frac{1}{\sqrt{2}} S_{2 z}+\sqrt{2} S_{2 x} S_{3 z}+\frac{1}{2} S_{3 z} \\
& \downarrow \quad(\pi / 4)_{-2 y}, \mathrm{PFG} \\
& \frac{1}{2}\left(S_{2 z}+2 S_{2 z} S_{3 z}+S_{3 z}\right) .
\end{aligned}
$$

The above deviation density matrix is equivalent to the traceless part of $\frac{1-\epsilon}{8} \mathbb{1}+\epsilon\left\{\frac{1}{2} \mathbb{1}_{S} \otimes|00\rangle\left\langle\left. 00\right|_{A}\right\}\right.$ where $\epsilon \sim 10^{-5}$ is the purity factor [20].

\section{Diagonal tomography:}

The diagonal tomography was carried out using a strong PFG to dephase out all the residual off-diagonal elements and using a $6^{\circ}$ non-selective linear readout pulse. The resulting intensities of the spectral lines constrain the diagonal elements $\left(d_{i}\right)$ of the traceless deviation density matrix. The experimental deviation density matrix is normalized w.r.t. the theoretical traceless density matrix such that they both have the same root mean square value $\sqrt{\sum_{i} d_{i}^{2}}$, and trace is introduced by adding identity matrix to the normalized deviation matrix. The resulting diagonal density matrix yields the probabilities. Estimation of random errors were carried out by several repetitions at each $\theta$ value. 